GRADIATION\&APPLICATIONS

ISSN 2466-4294 (online) | rad-journal.org

Vol. 2 | Issue 2 | pp. 115- 117, 2017

doi: 10.21175/RadJ.2017.02.024

Original research paper

\title{
THE EFFECT OF AIR FILTRATION ON THE FRACTION OF UNATTACHED RADON PRODUCTS ${ }^{*}$
}

\author{
Janja Vaupotič $\check{c}^{* *}$, Mateja Bezek, Ivan Kobal \\ Department of Environmental Sciences, Jožef Stefan Institute, Ljubljana, Slovenia
}

\begin{abstract}
An air cleaner was installed in a room with elevated radon activity concentration, and the following parameters have been monitored: activity concentrations of ${ }^{222} R n(R n)$ and its short-lived products (RnP), degree of equilibrium between $R n$ and $R n P(F)$, fraction of unattached $R n P$ (fun), and the number concentration and size distribution of aerosol particles (5-530 nm). Several hours of filtration removed the $>10 \mathrm{~nm}$ particles almost completely, thus increasing the contribution of the $<10 \mathrm{~nm}$ particles, associated with unattached RnP. Consequently, fun was substantially augmented with a concomitant decrease in $F$.
\end{abstract}

Key words: Air filtration, radon, unattached radon products

\section{INTRODUCTION}

Radioactive noble gas radon $\left({ }^{222} \mathrm{Rn}\right)$, a product of ${ }^{226} \mathrm{Ra} \alpha$-transformation in the ${ }^{238} \mathrm{U}$ radioactive series, is present, at lower or higher level, in any environmental medium. Its $\alpha$-transformation starts a sequence of radioactive transformations, giving birth to the radon short-lived products (RnP): ${ }^{218} \mathrm{Po} \rightarrow{ }^{214} \mathrm{~Pb} \rightarrow{ }^{214} \mathrm{Bi} \rightarrow$ 214Po. Initially, they are positive ions, which in air are soon neutralised by interactions with air molecules, mostly water traces, and form molecular clusters, with activity median diameter no larger than $30 \mathrm{~nm}$ and known as unattached $\mathrm{RnP}$ [1]. In addition, RnP ions, atoms and clusters also partly attach to aerosol nano particles and form the so-called attached RnP (size range 50-500 $\mathrm{nm}$ ). Therefore, dynamics of $\mathrm{RnP}$ in air is closely related to the dynamics of background aerosol, and both the extent of equilibrium between $\mathrm{Rn}$ and $\operatorname{RnP}(F)$ and fraction of unattached $\operatorname{RnP}\left(f_{\text {un }}\right)$, two key input data in radon dosimetry [2], are governed by concentration and size distribution of aerosol particles in indoor air.

Radon and RnP have been recognised as contributing about half to the effective dose (radon contribution being minor) we receive from all natural radioactivity, and are a major cause of lung cancer, second only to smoking. Aerosol nano particles, in addition to being carrier of RnP into the respiratory tract, also show negative health effects. Therefore, levels of RnP and aerosols nano particles in dwellings and at workplaces should be kept acceptably low. One of the procedures to achieve this aim is ventilation, because it is a dominant parameter controlling $\mathrm{Rn}$ and RnP levels indoors [3]. Nevertheless, when applying this mitigation measure, cost-effectiveness should be borne in mind, as more fresh air requires enhanced heating in wintertime [4]. Low RnP levels can also be assured by air-conditioning [5], [6] and by air filtration using special filters [5], [7]-[9].

The latter is also the subject of the present work in which radon short-lived products and aerosols are monitored during air filtration using an air cleaner in a closed room. Results are presented and the impact of filtration on the fraction of unattached $\mathrm{RnP}$ and dose conversion factor are discussed.

\section{EXPERIMENTAL}

The experiments have been carried out during weekends in a basement playroom of a kindergarten (size $4 \mathrm{~m} \times 4 \mathrm{~m} \times 2.7 \mathrm{~m}$ ) with a weekly average radon activity concentration reaching up to $2500 \mathrm{~Bq} \mathrm{~m}^{-3}$. All instruments were placed in a corner, at a height of about $0.5 \mathrm{~m}$ above the floor and at a distance of $1.5 \mathrm{~m}$ from the single window opposite to the door.

Air was filtered with a $125 \mathrm{~W}$ mobile air cleaner designed to remove from air particles larger than 10 $\mathrm{nm}$. It uses a pre-filter and electrostatic filter and can be run at three airflow levels: 1: $300 \mathrm{~m}^{3} \mathrm{~h}^{-1}, 2: 700 \mathrm{~m}^{3}$ $\mathrm{h}^{-1}$, and 3: $1200 \mathrm{~m}^{3} \mathrm{~h}^{-1}$, the filtration rates $\left(\mathrm{h}^{-1}\right)$ of the room being 7,16 and 27, respectively.

Activity concentrations of radon $\left(A_{\mathrm{Rn}}\right)$ and its shortlived products (expressed by equilibrium-equivalent: $\left.A_{\mathrm{RnP}}=0.1065 A_{218 \mathrm{Po}}+0.515 A_{214 \mathrm{~Pb}}+0.379 A_{214 \mathrm{Bi}}\right)$, in the unattached ( $A_{\mathrm{RnP}}^{\mathrm{un}}$ ) and attached form ( $A_{\mathrm{RnP}}^{\text {att }}$, with: $\left.A_{\mathrm{RnP}}^{\mathrm{un}}+A_{\mathrm{RnP}}^{\mathrm{att}}=A_{\mathrm{RnP}}\right)$, degree of secular equilibrium

\footnotetext{
* The paper was presented at the Fifth International Conference on Radiation and Applications in Various Fields of Research (RAD 2017), Budva, Montenegro, 2017.

janja.vaupotic@ijs.si
} 
between Rn and RnP (equilibrium factor, $F=A_{\mathrm{RnP}}$ $\left./ A_{\mathrm{Rn}}\right)$, and fraction of unattached $\mathrm{RnP}\left(f_{\mathrm{un}}=A_{\mathrm{RnP}}^{\mathrm{un}} / A_{\mathrm{RnP}}\right)$ were obtained (once every two hours) using an EQF3020-2 device (Equilibrium Factor Monitor, Sarad, Germany).

Number concentration and size distribution of aerosol particles in the $5-530 \mathrm{~nm}$ size range were measured with an SMPS+C instrument (Scanning Mobility Particle Sizer + Counter), Series 5.400, with the medium DMA (Differential Mobility Analyser) unit (Grimm, Germany). The instrument gives (every four minutes) the total number concentration of particles $\left(N_{\mathrm{t}}\right)$ in the above size range, particle number concentrations in each of the 44 size windows $\left(N_{\mathrm{d}}\right)$, particle number size distribution ( $\mathrm{d} N / \mathrm{d}$ lnd, with $d$ being the electrical mobility-equivalent particle diameter), and geometric mean of particle diameter (dGM).

\section{RESULTS AND DISCUSSION}

The results of several experiments carried out in one single day (on Saturday) are presented in Fig. 1. After the instruments were installed and put into operation in the evening prior to filtration, the room was left and closed, and was entered only briefly, first at about 8.00 a.m. to turn on the cleaner to level 1 (step 1), and then at 12.00 noon to increase the flow rate to level 2 (step 2), at 4.00 p.m. to switch to level 3 (step 3 ), and at 7.00 p.m. to turn off the cleaner (periods shaded in the plots).

The SMPS readings at 6.00 a.m. showed the total number concentration of aerosol nano particles $\left(N_{\mathrm{t}}\right)$ to be about $5000 \mathrm{~cm}^{-3}$ and geometric mean of their diameter $\left(d_{\mathrm{GM}}\right)$, about $70 \mathrm{~nm}$ (Fig. 1a), a common size outdoors [10]. In the particle size distribution (Fig. 2), particles in the 30-200 nm size were predominant, with the contribution of the $<10 \mathrm{~nm}$ particles being minor (as also evident from Fig. 1b), their concentrations being $4300 \mathrm{~cm}^{-3}$ and $50 \mathrm{~cm}^{-3}$, respectively.

As the filtration started at flow rate of $300 \mathrm{~m}^{3} \mathrm{~h}^{-1}$ in step 1, $N_{\mathrm{t}}$ decreased rapidly in about an hour and remained constant at $1200 \mathrm{~cm}^{-3}$ till the end of the step (Fig. 1a). $N_{30-200}$ showed a similar decrease, unless it continued to decrease slowly in the last hour (Fig. 1b). The low concentration of the $<10 \mathrm{~nm}$ particles was initially reduced, but started to grow in the second half of this step (Fig. 1b), obviously due to operation of the cleaner. The result of these changes is reflected in a $d_{\mathrm{GM}}$ decrease (Fig. 1a) and in the particle size distribution at the end of the step (Fig. 2).

In the first hour of step 2 at flow rate of $700 \mathrm{~m}^{3} \mathrm{~h}^{-1}$, the $<10 \mathrm{~nm}$ particles continued to be emitted and larger particles to be removed from air (Fig. 1b), resulting in a small $N_{\mathrm{t}}$ increase and additional $d_{\mathrm{GM}}$ decrease (Fig. 1a). After that, $N_{<10}$ and $N_{30-200}$ (and concomitantly $N_{\mathrm{t}}$ and $d_{\mathrm{GM}}$ ) remained constant and continued to do so also at flow rate $1200 \mathrm{~m}^{3} \mathrm{~h}^{-1}$ in step 3, although with bigger fluctuations (Figs. 1a and 1b), presumably due to a stronger air turbulence affecting particle dynamics [11].
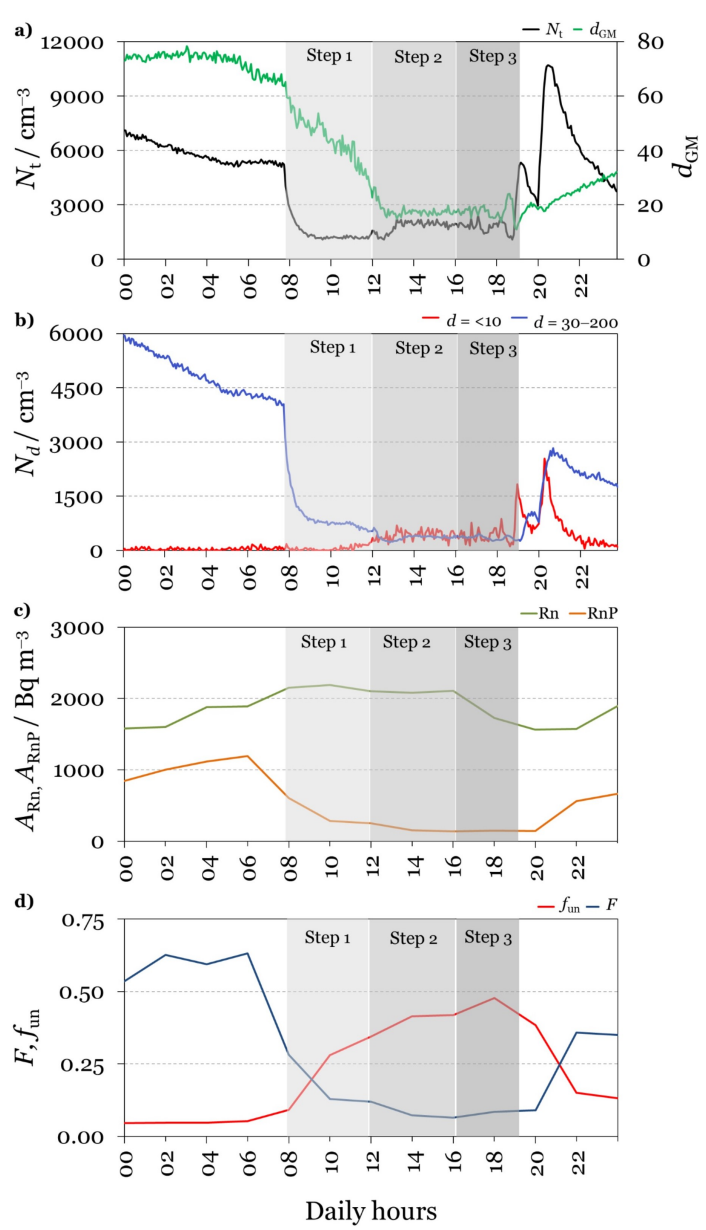

Figure 1. Time variations of: a) the total number concentration of aerosol nano particles $\left(N_{\mathrm{t}}\right)$ and geometric mean of their diameters $\left.\left(d_{\mathrm{GM}}\right), \mathrm{b}\right)$ number concentration of aerosol particles smaller than $10 \mathrm{~nm}\left(N_{<10}\right)$ and particles in the 30-200 nm size range $\left(N_{30-200}\right)$, c) activity concentration of radon $\left(A_{\mathrm{Rn}}\right)$ and activity concentration of radon short-lived products $\left(A_{\mathrm{RnP}}\right)$, and d) equilibrium factor $(F)$ and unattached fraction of radon short-lived products $\left(f_{\mathrm{un}}\right)$

As expected, effective removal of the 30-200 nm particles in step 1 (Fig. 1b) caused a marked decrease in $\mathrm{RnP}$ concentration (Fig. 1c), and hence in the equilibrium factor (Fig. 1d). On the other hand, the low concentration of the $<10 \mathrm{~nm}$ particles, related to the unattached RnP, not only remained constant but even increased (Fig. 1b), and thus enhanced $f_{\text {un }}$ (Fig. 1d), as already observed previously [5]. Because RnP generation and formation require time, there is an inherent time delay [8], [12] between changes in aerosol characteristics and changes in $f_{\text {un. Therefore, }}$ this impact cannot be observed during short events, such as smoking, candle burning or cooking, lasting usually less than an hour [13]. Our filtration lasted long enough [14], [15] to show $f_{\text {un }}$ increasing toward the end of filtration (Fig. 1d), although $N_{<10}$ did not change significantly after the middle of step 2 (Fig. 1b). 


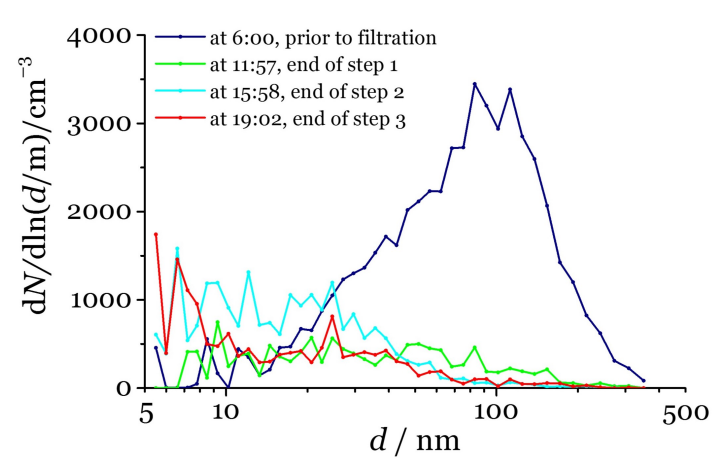

Figure 2. Number size distribution of aerosol nano particles prior to filtration (at 6:00), and during filtration at the end of step 1 (at 11:57), step 2 (at 15:58) and step 3 (at 19:02)

The equilibrium factor and unattached fraction are key quantities in discussing radon dose conversion factors in radon dosimetry [2]. According to Porstendörfer [1], dose conversion factor $f_{\mathrm{DC}}$ for nasal breathing (light work) can be obtained using the empirical equation: $f_{\mathrm{DC}}=23 f_{\mathrm{un}}+6.2\left(1-f_{\mathrm{un}}\right)$. Our value $f_{\text {un }}=0.09$ prior to filtration gives $f_{\mathrm{DC}}=7.7 \mathrm{mSv}$ $\mathrm{WLM}^{-1}$, and $f_{\mathrm{un}}=0.48$ at the end of filtration gives $f_{\mathrm{DC}}$ $=14.3 \mathrm{mSv} \mathrm{WLM}^{-1}$, thus increased by about twice. On other hand, during this time $A$ RnP decreased from 1200 $\mathrm{Bq} \mathrm{m}-3$ to $200 \mathrm{~Bq} \mathrm{~m}^{-3}$, by a factor of 6 . Thus, filtration reduced the effective dose substantially, by about three times; even more than reported earlier [7].

\section{CONCLUSIONS}

Running an air cleaner for several hours at a flow rate of $700-1200 \mathrm{~m}^{3} \mathrm{~h}^{-1}$ in a $45 \mathrm{~m}^{3}$ closed room caused a decrease of the number concentration of aerosol particles greater than $30 \mathrm{~nm}$ from $4300 \mathrm{~cm}^{-3}$ to 300 $\mathrm{cm}^{-3}$ and decrease in the activity concentration of radon short-lived products from $1200 \mathrm{~Bq} \mathrm{~m}^{-3}$ to 200 $\mathrm{Bq} \mathrm{m}^{-3}$ (with radon concentration remaining unchanged) and equilibrium factor from 0.60 to 0.10 . Concomitantly, fraction of unattached radon products rose from 0.09 to 0.48 , thus increasing the dose conversion factor by a factor of about 2 . As a result of filtration, the exposure to nano aerosol was substantially decreased and the rate of the committed effective dose was reduced by about a factor of 3 .

Acknowledgement: The authors acknowledge the financial support from the Slovenian Research Agency (research core funding No. P1-O143).

\section{REFERENCES}

1. J. Porstendörfer, "Radon: measurements related to dose," Environ. Int., vol. 22, no. 1, pp. S563-S583, Jun. 1996. DOI: 10.1016/So16o-4120(96)oo158-4

2. J. D. Harrisson and J. W. Marsh, "Effective dose from inhaled radon and its progeny," in Proc. First ICRP Symp. on the International System of Radiological Protection, Bethesda (MD), USA, 2012, pp. 378-388.

DOI: 10.1016/j.icrp.2012.06.012
3. G. V. Ashok et al., "Indoor radon concentration and its possible dependence on ventilation rate and flooring type," Radiat. Prot. Dosim., vol. 148, no. 1, pp. 92-100, Feb. 2012.

DOI: $10.1093 / \mathrm{rpd} / \mathrm{ncq} 590$

PMid: 21335628

4. T. Katona et al., "Cost assessment of ventilation and averted dose due to radon in dwellings," J. Environ. Radioact., vol. 79, no. 2, pp. 223-223, 2005.

DOI: 10.1016/j.jenvrad.2004.07.004 PMid: 15603909

5. S. Tokonami et al., "Changes in indoor aerosol characteristics and their associated variation on the dose conversion factor due to radon progeny inhalation," Radioisotopes, vol. 52, no. 6, pp. 285-292, Jan. 2003.

DOI: 10.3769/radioisotopes.52.285

6. D. Grządziel et al., "The influence of air conditioning changes on the effective doses due to radon and its short-lived decay products," Nukleonika, vol. 61, no. 3, pp. 239-244, Sep. 2016. DOI: 10.1515/nuka-2016-0040

7. Y. Yasuoka et al., "Radon mitigation using an air cleaner," J. Radioanal. Nucl. Chem., vol. 279, no. 3, pp. 885-891, Mar. 2009. DOI: $10.1007 / \mathrm{s} 10967-008-7379-0$

8. J. Wang et al., "Mitigation of radon and thoron decay products by filtration," Sci. Total Environ., vol. 409, no. 19, pp. 3613-3619, Sep. 2011.

DOI: $10.1016 /$ j.scitotenv.2011.06.030 PMid: 21767867

9. K. Iwaoka et al., "Mitigation effects of radon decay products by air cleaner," J. Radioanal. Nucl. Chem., vol. 295, no. 1, pp. 639-642, Jan. 2013. DOI: $10.1007 /$ s10967-012-1813-z

10. J. Vaupotič, "Nano particles including radon decay products in ambient air," in Chemistry, Emission Control, Radioactive Pollution and Indoor Air Quality, N. Mazzeo, Ed., Rijeka, Croiatia: InTech, 2011, ch. 6, pp. $153-190$ DOI: $10.5772 / 16369$

11. N. Stevanovic et al., "Deposition rates of unattached and attached radon progeny in room with turbulent airflow and ventilation," J. Environ. Radioact. vol. 100, no. 7, pp. 585-589, Jul. 2009.

DOI: 10.1016/j.jenvrad.2009.04.007 PMid: 19428163

12. D. Nikolopoulos and E. Vogiannis, "Modelling radon progeny concentration variations in thermal spas," Sci. Total Environ., vol. 373, no. 1, pp. 82-93, Feb. 2007.

DOI: 10.1016/j.scitotenv.2006.11.017 PMid: 17188335

13. M. Smerajec and J. Vaupotič, "Nanoaerosols including radon decay products in outdoor and indoor air at a suburban site," J. Toxicol., vol. 2012, p. 510876 , Feb. 2012.

DOI: $10.1155 / 2012 / 510876$

PMid: 22523488 PMCid: PMC 3317232

14. M. Bezek et al., "Radon decay products and 10-1100 nm aerosol particles in Postojna Cave," Nat. Hazards Earth Syst. Sci., vol. 13, no. 3, pp. 823-831, Mar. 2013. DOI: 10.5194/nhess-13-823-2013

15. C. Vargas Trassierra et al., "Effect of indoor-generated airborne particles on radon progeny dynamics," J. Hazard. Mater., vol. 314, pp. 155-163, Aug. 2016 DOI: 10.1016/j.jhazmat.2016.04.051

PMid: 27131455 